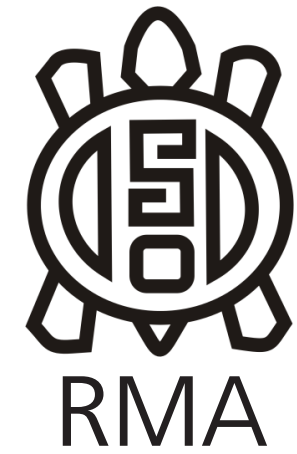

Dossier

\title{
Aportes al estudio de los conjuntos líticos recuperados en áreas de aprovisionamiento de rocas: formas de abordaje desde el análisis tecno-tipológico
}

\author{
Contributions to the study of the lithic assemblages recovered in rocks \\ procurement areas: approach from techno-typological analysis
}

\author{
Federico Miguel Bobillo*, María Laura Salgán**, Gisela Sario*** \\ * Instituto Superior de Estudios Sociales (CONICET). E-mail: fede_bobillo@yahoo.como.ar \\ **Instituto de Evolución, Ecología Histórica y Ambiente (CONICET- \\ UTN FRSR); International Center for Earth Science, Malargüe, Mendoza, Argentina. \\ E-mail: Isalgan@mendoza-conicet.gob.ar \\ *** Instituto de Antropología de Córdoba (CONICET) y Museo de \\ Antropología, FFyH, UNC. E-mail: giselasario@hotmail.com
}

\begin{abstract}
La presentación de este simposio se realizó en el marco del Primer Congreso Argentino de Estudios Líticos en Arqueología ( $1^{\circ}$ CAELA). El mismo se llevó a cabo en la provincia de Córdoba (Argentina) entre los días 19 y 22 de septiembre de 2018 y contó con el apoyo de distintas instituciones académicas; entre las que se encuentran: Facultad de Filosofía y Humanidades-Universidad Nacional de Córdoba, CONICET, Instituto de Antropología de Córdoba (IDACOR-CONICET/ UNC), Museo de Antropología (FFyH-UNC), Laboratorio de análisis macro y microscópico de materiales líticos (LAMMAL CONICET) y el Programa de Arqueología Digital (IDACORMuseo de Antropología, UNC), entre otros.
\end{abstract}

El interés que motivó la realización del $1^{\circ}$ CAELA fue la posibilidad de reunir a investigadores que trabajan distintos temas relacionados con el análisis lítico en Argentina. En el marco de este espacio de encuentro se logró poner en común y discutir problemáticas relacionadas con el estudio de la tecnología lítica, como así también el conjunto de metodologías y técnicas que actualmente se utilizan en los equipos de investigación de distintas regiones del país.

Particularmente, el simposio "El estudio de conjuntos líticos en las áreas de aprovisionamiento: variabilidad y formas de abordaje desde el análisis tecno-tipológico" tuvo por objeto generar un ámbito de intercambio de información y experiencias sobre distintos casos de estudio vinculados con el estudio de las áreas de aprovisionamiento y las metodologías utilizadas para analizar el registro arqueológico presente en dichas áreas. Este simposio, como un espacio de puesta en común entre colegas, permitió integrar ideas e información referida al análisis tecno-tipológico de los materiales líticos de canteras y canteras-taller, y su potencialidad como fuente de información. Además, se discutieron distintos métodos y técnicas como vía de resolución de diferentes problemáticas relacionadas con el estudio de las áreas de aprovisionamiento. Se expusieron trece trabajos de investigación, que permitieron destacar la relevancia y calidad de los aportes de los estudios líticos en las áreas de aprovisionamiento, y su aporte a la arqueología Argentina. En el presente Dossier se compilan siete de estos aportes.
El trabajo de Silvina Castro, Lucía Yebra, Valeria Cortegoso, Gustavo Lucero, Víctor Durán, Erik Marsh y Diego Winocur, titulado INVESTIGACIONES GEOARQUEOLÓGICAS DE FUENTES LITICAS EN LOS ANDES ARGENTINOS: GÉNESIS DE LAS ROCAS, COSTOS DE ACCESIBILIDAD Y SISTEMAS DE PRODUCCIÓN LÍTICA, aborda el caso particular de las canteras líticas ubicadas en ambientes de altura de Mendoza y San Juan. El objetivo de este trabajo fue comprender la génesis de las rocas, los modos de aprovisionamiento y los sistemas de producción lítica utilizando una perspectiva geoarqueológica. Para ello, los autores aportan información sobre la base regional de recursos líticos y analizan los procesos de talla en distintas escalas espaciales. Utilizan la herramienta de análisis espacial SIG, con el fin de evaluar la accesibilidad a las fuentes, el costo de traslado, la distribución espacial de las muestras arqueológicas y el acceso a las áreas de estudio. También se llevaron a cabo estudios tecnológicos desde una perspectiva organizacional y sistémica, y se efectuaron análisis macroscópicos y petrográficos de muestras. A partir de esta investigación, los autores discutieron los cambios y continuidades en el uso de recursos líticos en relación a las estrategias de subsistencia y movilidad. Esta información, en conjunto con los datos obtenidos del análisis tecnológico, permitieron indagar sobre los cambios en las estrategias humanas desde distintas escalas espaciales.

En el trabajo de Mariángeles Borgo, Guillermo Heider, Gabriel Ramos y Rafael Curtoni, titulado ESTUDIOS PRELIMINARES EN EL ÁREA DE CANTERAS LA FALLA (SAN LUIS), se presenta el estudio sobre las canteras arqueológicas y las fuentes potenciales de materias primas líticas procedentes de la cuenca del río Quinto (centro-este de la provincia de San Luis). El objetivo es indagar en las estrategias de aprovisionamiento y movilidad implementadas por los grupos que habitaron el área durante todo el Holoceno, a partir del estudio de la organización de la tecnología y la confección de una base regional de recursos líticos. El trabajo de estos autores en un sitio permitió reconocer las características generales de gestión tecnológica en la que se combinan estrategias expeditivas y conservadas, actividades de reducción lítica y su distribución. 
El estudio realizado por Natalia Cirigliano, M. Cecilia Pallo y Judith Charlin, titulado EXPLOTACIÓN Y CIRCULACIÓN DE ROCAS DE GRANO FINO OSCURAS TIPO POTROK AIKE: EL ANÁLISIS DE DOS CONJUNTOS LÍTICOS DE PATAGONIA EXTREMO SUR CONTINENTAL (ARGENTINA), tiene por objeto analizar la abundancia, las características tecno-morfológicas y la intensidad de uso de artefactos confeccionados en Rocas de Grano Fino Oscuras tipo Potrok Aike (RGFO PKA) de Patagonia extremo sur continental. En este sentido, se busca conocer como los grupos de cazadores-recolectores ocuparon el espacio y efectuaron la explotación de recursos en el pasado. Para ello, las autoras realizan una caracterización de la disponibilidad de RGFO PKA, de la distribución de artefactos y un análisis tecno-morfológico. A partir de este trabajo se identificaron conjuntos localizados a diferentes distancias del área de captación de RGFO PKA. En base a los datos obtenidos, se postula que habría existido una adquisición directa de materia prima, cuyo transporte se habría dado bajo la forma de nódulos o núcleos. A partir de esta investigación, recabaron información novedosa sobre la disponibilidad y características de los nódulos de RGFO PKA y, a la vez, generar expectativas sobre el modo de explotación y la forma de transporte por parte de los grupos cazadores-recolectores en el pasado.

En la contribución PRODUCCIÓN LÍTICA EN CANTERAS-TALLER DE ANTOFAGASTA DE LA SIERRA (CATAMARCA). UN APORTE A LA COMPRENSIÓN DEL REGISTRO LÍTICO Y SU DIVERSIDAD TECNO-TIPOLÓGICA, Federico Bobillo realiza un estudio de las prácticas y saberes desplegados por los artesanos en canterastaller, y la variabilidad tecno-tipológica que estos distintos saberes y modos de trabajar las rocas generaron durante miles de años de explotación de recursos líticos. A través de este estudio, se logra registrar una diversidad tecno-tipológica que se atribuye a distintos procedimientos y métodos para reducir núcleos y extraer formas-base. También se reconocen desechos de talla con atributos técnico-morfológicos particulares que dan cuenta de acciones específicas en la talla de lascas nodulares, y grupos tipológicos que expresan un corpus de saberesprácticos distintivo. A partir de este trabajo, el autor accede a la variabilidad de materiales líticos producto de distintas prácticas y operaciones que intervienen en la dinámica tecnológica.

Lucas Vetrisano, Nora Franco y Pablo Bianchi, en el ANÁLISIS DE UNA CANTERA-TALLER DE MADERA SILICIFICADA EN EL EXTREMO-SUR DEL MACIZO DEL DESEADO (PATAGONIA, ARGENTINA), proponen analizar una fuente primaria ubicada en el extremo sur del Macizo del Deseado, cuyas primeras evidencias de utilización se registran hacia el 8000 AP. Su objetivo es evaluar si las características que presentan la madera silicificada en el terreno, condiciona de alguna manera su aprovechamiento por parte de las poblaciones humanas. Abordan una muestra artefactual de origen superficial de una fuente primaria, donde ponen especial énfasis en el análisis de las primeras etapas de reducción. Proponen discutir la premisa que sostiene que el desbaste de los troncos fosilizados se realiza siguiendo el sentido de las vetas, lo que resulta en que los productos del desbaste alcancen proporciones laminares. El conjunto analizado fue obtenido por medio de un muestreo superficial, donde evaluaron la posición del eje técnico en relación a las vetas y las posibles variaciones en el método de talla en relación al grado de silicificación de los troncos. Los resultados les permiten sostener que hay un aprovechamiento diferencial de los troncos en relación con su grado de silicificación y que las extracciones no están condicionadas a la calidad de talla de la materia prima. Por lo que concluyen que el aumento de laminaridad registrado en el Holoceno medio en el Macizo del Deseado, parece no responder a una restricción técnica de esta materia prima.

Por otra parte, María Cecilia Pallo, Natalia Andrea Cirigliano, Judith Charlin y Karen Borrazzo, realizan UNA APROXIMACIÓN A LA DISTRIBUCIÓN DE LA PRODUCCIÓN LAMINAR EN PATAGONIA MERIDIONAL. Las autoras analizan la distribución de la producción laminar manufacturada en lutita, al sur de la Sierra Baguales. Analizan el potencial de la presencia de láminas en baja frecuencia, como un posible marcador arqueológico de conexiones espaciales y circulación de bienes, que podría involucrar mecanismos de interacción poblacional y/o la superposición de distintos rangos de acción. Como un primer acercamiento a esta discusión, evalúan la existencia de fuentes potenciales de aprovisionamiento de lutita, considerando la distribución, tamaño e intensidad de uso de estos artefactos, en función de la distancia a las fuentes potenciales. Los resultados indican que las fuentes potenciales de lutita, permiten explicar la distribución de los artefactos en el área, aunque aún no se registró la fuente de explotación efectiva. Destacan la necesidad de futuros estudios petrográficos y análisis de rayos $X$ para ajustar la caracterización de la/s fuente/s potencial/es de procedencia de la roca empleada y descartar posible confusión con otras materias primas (e.g. pizzaras). Así mismo proponen como agenda futura revisar colecciones de artefactos y tomar otras medidas de intensidad de uso, para establecer comparaciones más precisas sobre la producción laminar.

Por último, en el trabajo LAS PALAS/AZADAS Y/O CUÑAS LÍTICAS DE CERRO EL PECEÑO (SUR DE MENDOZA): UNA PRIMERA APROXIMACIÓN TECNOLÓGICA, María Laura Salgán y María de la Paz Pompei, analizan un conjunto de herramientas con morfología de palas/azadas y/o cuñas, recuperadas en el contexto de una fuente primaria de obsidiana, sin antecedentes previos en el sur de Mendoza. Las autoras discuten si los artefactos trabajados en pelita y dacita, dos materias primas que afloran en el lugar, pudieron estar vinculadas a las tareas de aprovisionamiento de obsidiana. Los resultados indican que en El Peceño estarían presentes todas las etapas de manufactura de estas herramientas, incluido su descarte. Proponen que su uso podría estar vinculado a las tareas de extracción de obsidiana, como cuñas o palancas para la extracción de materia prima con atributos específicos. Futuros análisis de huellas de uso y estudios funcionales ayudarán a discernir y contrarrestar las propuestas.

El estudio de los conjuntos líticos de canteras y canteras-taller es un tema que actualmente se encuentra en vías de desarrollo a nivel nacional e internacional. Por ello, agradecemos las presentaciones realizadas en el marco de este simposio, ya que contribuyen al conocimiento de temas, problemáticas y metodologías vinculadas con las áreas de aprovisionamiento de diferentes regiones del país. Como editores invitados de la Revista del Museo de Antropología de la Universidad de Córdoba, agradecemos el compromiso de los autores y del comité organizador, por el esfuerzo realizado en concretar este dossier. 